\title{
Determination of elastic resonance frequencies and eigenmodes using the method of fundamental solutions
}

\author{
Carlos J.S. Alves ${ }^{\mathrm{a}, *}$, Pedro R.S. Antunes ${ }^{\mathrm{b}}$ \\ ${ }^{a}$ CEMAT-IST, Universidade de Lisboa, Av. Rovisco Pais 1, Lisbon 1049-001, Portugal \\ ${ }^{\mathrm{b}}$ GFM-FCUL, Universidade de Lisboa, Campo Grande C6, Lisboa 1749-016, Portugal
}

\section{A R T I C L E I N F O}

\section{Keywords:}

Elastic wave scattering

Meshfree method

Method of fundamental solutions

Resonance frequencies

\begin{abstract}
A B S T R A C T
In this paper, we present the method of fundamental solutions applied to the determination of elastic resonance frequencies and associated eigenmodes. The method uses the fundamental solution tensor of the Navier equations of elastodynamics in an isotropic material. The applicability of the the method is justified in terms of density results. The accuracy of the method is illustrated through 2D numerical examples for the disk and non trivial shapes.
\end{abstract}

\section{Introduction}

In this work we extend the method of fundamental solutions (MFS) to the calculation of elastic resonance frequencies and implement the method to find the associated eigenmodes and nodal lines for the disk and some other non trivial simply connected domains in 2D. In mechanical or civil engineering applications the knowledge of resonance frequencies and natural eigenmodes is of great importance as it indicates the possible failure of a structure due to resonance phenomena.

The MFS was introduced for the calculation of eigenvalues of the Laplacian in 2001 with the work of Karageorghis [18]. The application of the MFS for the calculation of resonance frequencies, eigenmodes and its nodal domains was then considered for more general shapes in [2] proving adequate density results for the acoustic case [2,4]. A similar technique was used for the calculation of the eigenvalues and eigenmodes of the bi-Laplacian operator in [3], which is an approach to search vibrational characteristics of thin elastic plates (e.g. [24]).

Although the MFS has been used for direct or inverse elastic problems (e.g. [5-7,11,19,25,27,29]), to our knowledge it has not yet been used for the calculation of elastic resonance eigenmodes in a bounded domain. In fact, even with other types of numerical methods, there seems to be little research is this area and the 1985 book by Kitahara [20] is still a useful reference - in that case using boundary integral methods.

There are some references that deal with an associated eigenvalue problem for the bi-Laplacian, or thin shells, such as $[3,14,16,24,28]$, and the subject is present in related vibrodynamic problems, such as aircraft vibrations or elastic shells (e.g. [15,22,31]). We will not consider here the exterior problem, where complex resonance frequencies appear in scattering problems with arbitrary obstacles, that may be close to real axis, with trapping obstacles (e.g. [1,13]).

Here we will use the fundamental solutions of the elastodynamics Navier equation and although density results have been considered for these fundamental solutions (cf. [6,7]), the resonance problem demands that a previous restriction to non resonance frequencies should be avoided. This is of particular importance to justify the calculation of the eigenmodes. Therefore in Section 3 we derive appropriate density results that justify the algorithmic approach set in Section 4.

Finally in Section 5 we present different numerical experiments for several 2D simply connected shapes. The algorithm is first tested in terms of convergence and ill conditioning, leading to a particular choice of source points, as proposed in [2]. For simple shapes, such as the disk, we are able to obtain errors of machine precision magnitude. We also present numerical results that compare the evolution of the resonance frequencies in terms of the Poisson coefficient and consider other non trivial shapes, with excellent results. It should be noted that here the eigenmodes have 2 components and the shapes of each component eigenmode may differ substantially, presenting different nodal shapes. These are fully illustrated in the examples we present.

\section{Elastic waves in isotropic bounded domains}

We consider a regular bounded simply connected domain $\Omega \subset \mathbb{R}^{D}$ with piecewise $C^{1}$ boundary $\Gamma=\partial \Omega$, in dimensions $D=2,3$. The linear elastic Lamé operator will be here denoted by $\Delta^{\star}=\nabla \cdot \nabla^{\star}$, where $\nabla^{\star}$ is the stress tensor, given by

$$
\nabla^{\star}(u)=(\lambda \nabla \cdot u) \mathbf{I}+\mu\left(\nabla u+\nabla u^{\top}\right),
$$

\footnotetext{
* Corresponding author.

E-mail addresses: carlos.alves@math.tecnico.ulisboa.pt (C.J.S. Alves), prantunes@ciencias.ulisboa.pt (P.R.S. Antunes).
} 


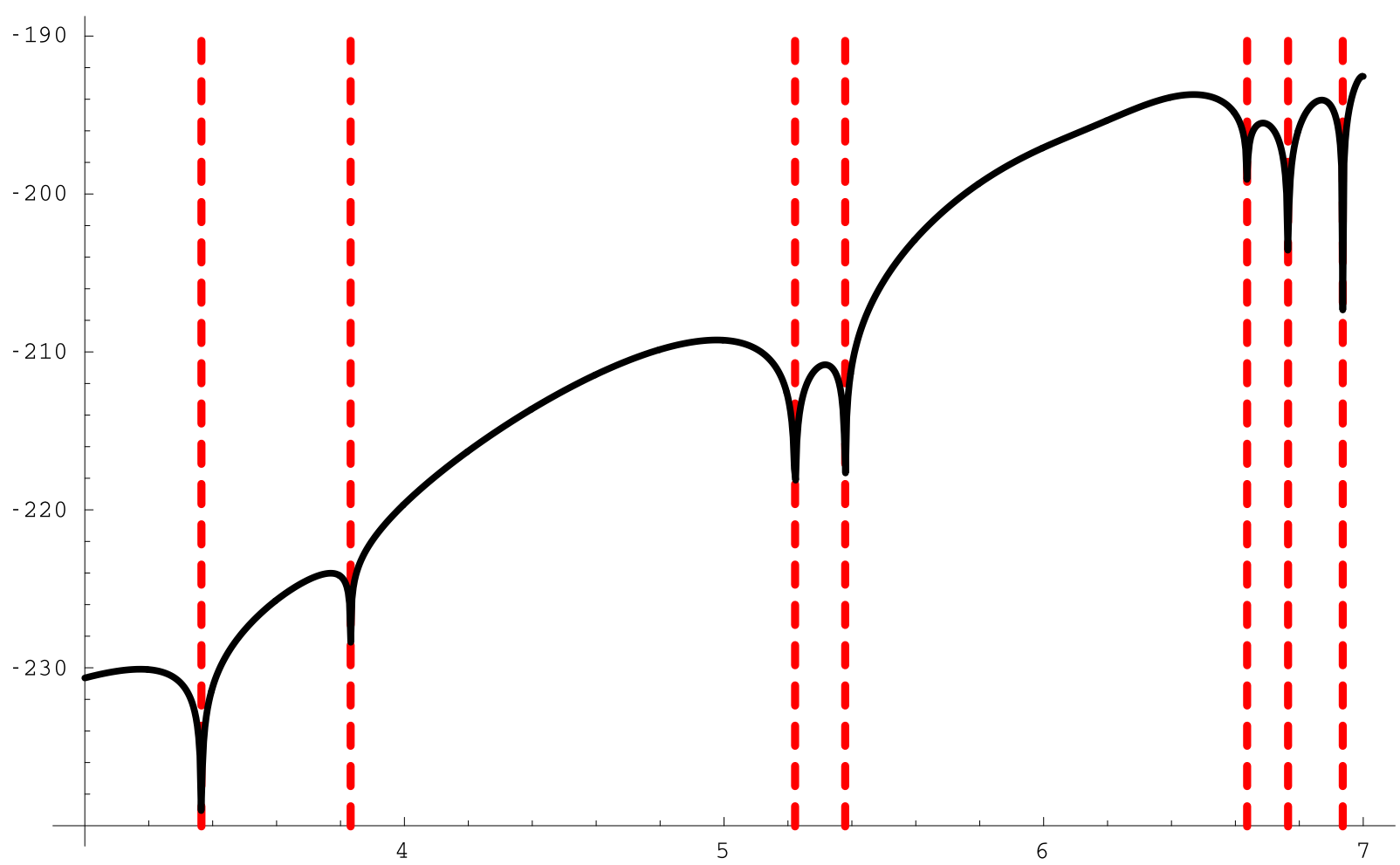

Fig. 5.1. Logarithm of the absolute value of the determinant of the matrix, as a function of $\omega$. The dashed red lines correspond to the exact eigenfrequencies.

where $\lambda, \mu>0$ are the Lamé parameters and $\mathbf{I}$ is the $D \times D$ identity matrix.

Given a constant frequency $\omega>0$, the time harmonic elastic waves satisfy the Navier equation (e.g. $[20,21]$ ),

$\Delta^{\star} u+\rho \omega^{2} u=\mu \Delta u+(\lambda+\mu) \nabla \nabla \cdot u+\rho \omega^{2} u=0$,

where $\rho$ denotes the variable density of the elastic material, that we will often assume to be unitary $(\rho=1)$.

The scattered wave $u$ in $\Omega$, induced by an incident elastic wave $u^{\text {inc }}$ is given by the boundary value problem

$\begin{cases}\Delta^{\star} u+\omega^{2} u=0 & \text { in } \Omega \\ \mathcal{B}\left(u+u^{i n c}\right)=0 & \text { on } \Gamma\end{cases}$

where $B$ denotes the boundary differential operator, which is the identity trace $B u=\left.u\right|_{\Gamma}$ for the Dirichlet problem or the normal trace $B u=$ $\left.\left(\partial_{\mathrm{n}}^{\star} u\right)\right|_{\Gamma}$ for the Neumann problem, with $\partial_{\mathrm{n}}^{\star} u=\nabla^{\star}(u)$ n, which is the normal stress tensor. Here $n$ denotes the unit normal vector on $\Gamma$, pointing outwards with respect to $\Omega$.

Problem (2.3) is well-posed provided $-\omega^{2}$ is not an eigenvalue of the Lamé operator $\Delta^{\star}$ in $\Omega$ (e.g. [21,26]).

In this paper we will be interested in using the MFS to obtain these eigenvalues, and in this case $\omega$ is also called a resonance frequency. We will first address the theoretical problem that consists in finding the resonance frequencies $\omega$ for which there exists an eigenmode $u \neq 0$ such that

$\begin{cases}\Delta^{\star} u+\omega^{2} u=0 & \text { in } \Omega \\ B u=0 & \text { on } \Gamma\end{cases}$

and that this eigenmode can be expressed as a linear combination of fundamental solutions

$u(x)=\sum_{j=1}^{\infty} \mathscr{G}_{\omega}\left(\left|x-y_{j}\right|\right) \boldsymbol{a}_{j}$ where the fundamental solution $\mathscr{G}_{\omega}$ will be defined in (3.4) and where $y_{j}$ are point sources outside $\bar{\Omega}$, and $\boldsymbol{a}_{j}$ are the respective vectorial weights to be determined by the MFS system.

\section{Density results with fundamental solutions}

The fundamental solution of the scalar Helmholtz operator $\Delta+\kappa^{2}$ (where $\Delta$ stands for the standard Laplace operator) is given in dimension $D=2$ by

$\Phi_{\kappa}(r)=\frac{i}{4} H_{0}^{(1)}(\kappa r)$,

where $r=|x|$ is the radial distance and $H_{0}^{(1)}=J_{0}+i Y_{0}$ denotes the Hänkel function defined with Bessel functions $J_{0}$ and $Y_{0}$. In dimension $D=3$ the fundamental solution is given by

$\Phi_{\kappa}(r)=\frac{\exp (i \kappa r)}{4 \pi r}$.

From its definition $\Phi_{\kappa}$ satisfies $-\left(\Delta+\kappa^{2}\right) \Phi_{\kappa}=\delta$, where $\delta$ is the Dirac delta distribution.

In elasticity there are two wavenumbers associated with the decomposition of $u$ into pressure $(P)$ and shear $(S)$ waves,

$\kappa_{S}=\omega \sqrt{\frac{\rho}{\mu}}, \quad \kappa_{P}=\omega \sqrt{\frac{\rho}{\lambda+2 \mu}}$.

The fundamental solution of the Navier operator $\Delta^{\star}+\omega^{2} \mathbf{I}$ is given by the symmetric Kupradze tensor (e.g. $[7,21]$ )

$\mathscr{G}_{\omega}=\frac{1}{\rho \omega^{2}}\left(\kappa_{S}^{2} \Phi_{\kappa_{S}} \mathbf{I}+\mathscr{D}\left(\Phi_{\kappa_{S}}-\Phi_{\kappa_{P}}\right)\right)$,

where $\mathbf{I}$ is the identity matrix and $\mathscr{D}=\left[\partial_{i j}^{2}\right]$ is the second derivative Hessian tensor. The fundamental solution equation is then satisfied,

$-\left(\Delta^{\star}+\omega^{2}\right) \mathscr{G}_{\omega}=\delta \mathbf{I}$,

where $\delta$ stands for the Dirac delta. 

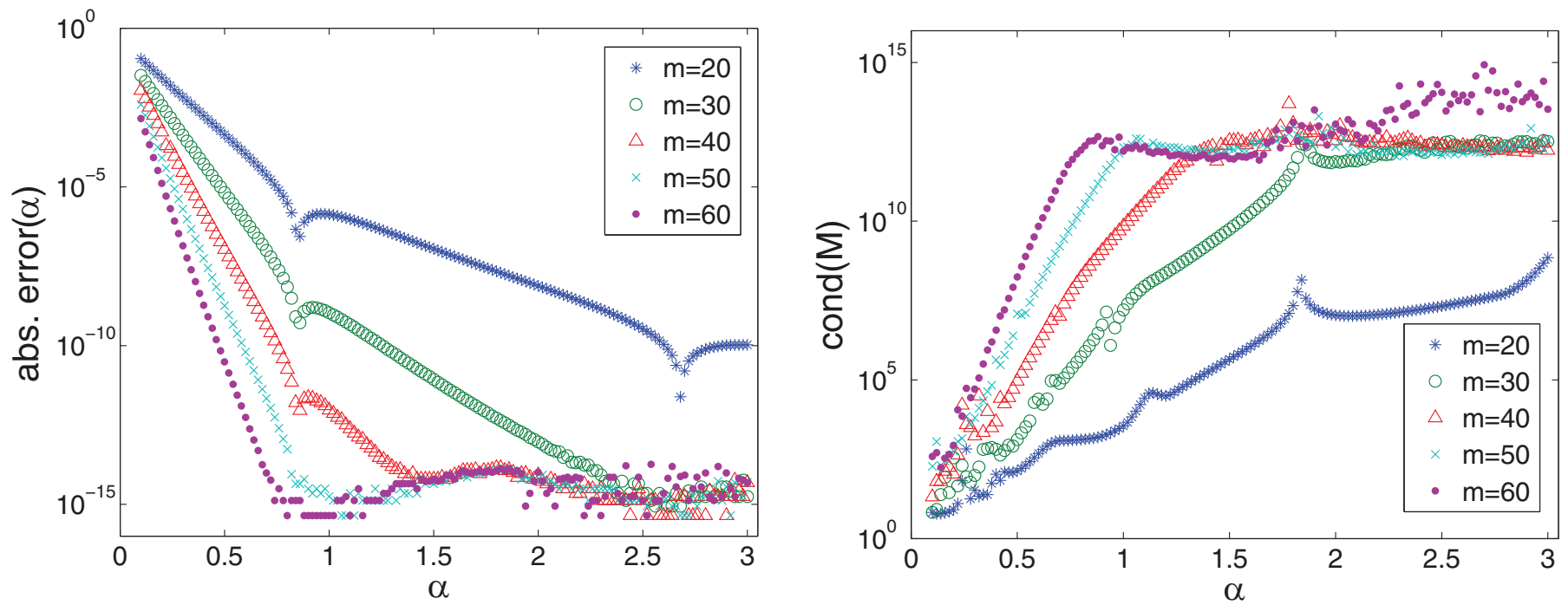

Fig. 5.2. Absolute errors and condition numbers as a function of $\alpha \in[0.1,3]$, for some values of $m$.
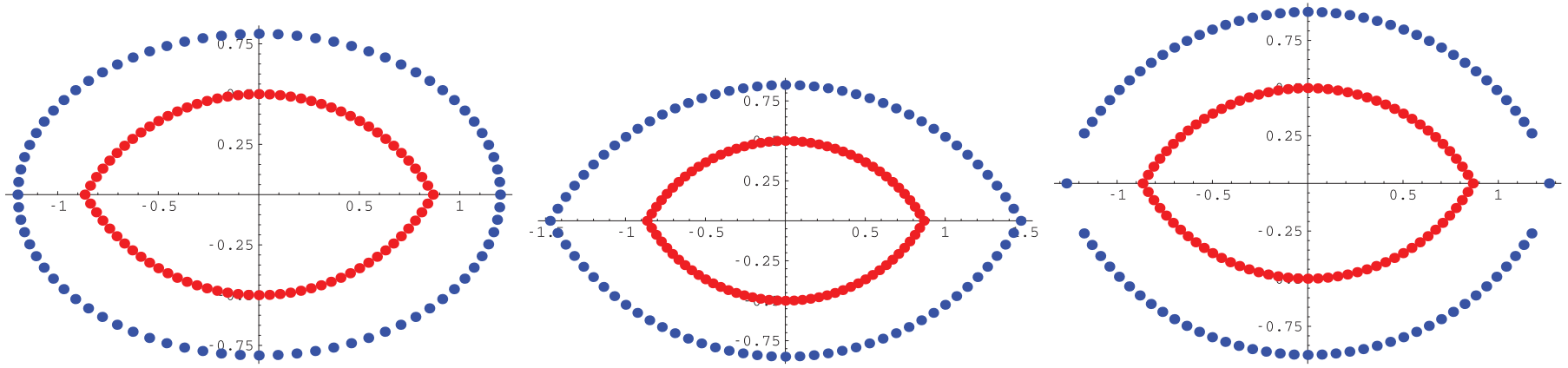

Fig. 5.3. The collocation points and three possible choices for the point-sources.
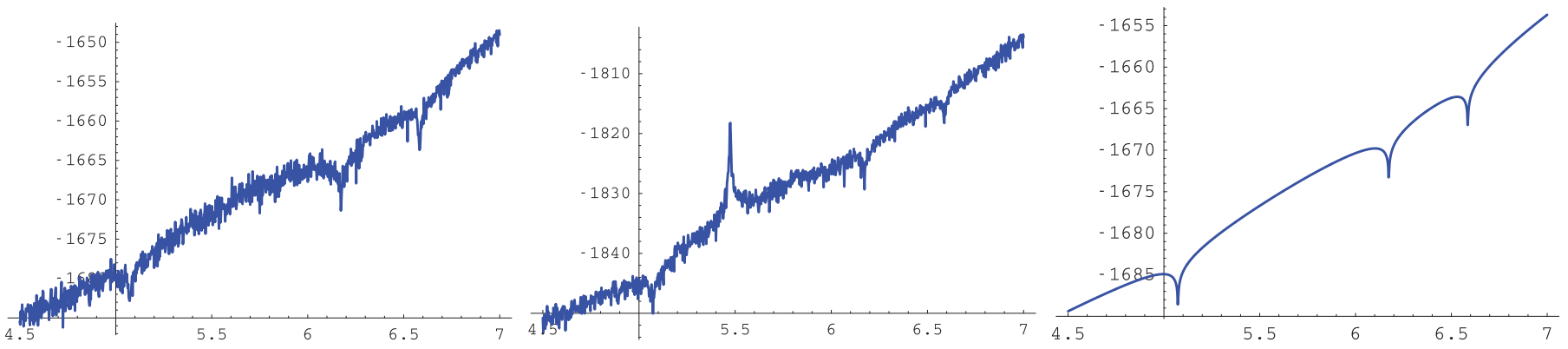

Fig. 5.4. The plots of $g(\omega)$, for the source points plotted in Fig. 5.3.

Definition 1. Taking a domain $\hat{\Omega} \supset \Omega$, we consider set of source points $\hat{\Gamma}_{m}=\left\{y_{1}, \ldots, y_{m}\right\} \in \hat{\Gamma}=\partial \hat{\Omega}$ and define

$S_{\omega}\left(\hat{\Gamma}_{m}\right)=\left\{\sum_{j=1}^{m} \mathscr{G}_{\omega}\left(\left|x-y_{j}\right|\right) \boldsymbol{a}_{j}: \boldsymbol{a}_{j} \in \mathbb{C}^{D}\right\}$.

We also define $S_{\omega}(\hat{\Gamma})$ in the limiting case of $S_{\omega}\left(\hat{\Gamma}_{m}\right)$, when the set $\hat{\Gamma}_{m}$ becomes dense in $\hat{\Gamma}$.

Density results have been established (see [7]) assuming non resonance frequencies. However in this case we want to find the resonance frequencies so that this assumption is avoided.
A density result will here be proved for any frequency $\omega \in \mathbb{R}$, in the space of Navier solutions $\mathscr{H}_{\omega}$.

Definition 2. We define the space of solutions of the Navier equation in $\Omega$ to be

$\mathscr{H}_{\omega}=\left\{u \in\left[H^{1}(\Omega)\right]^{D}:\left(\Delta^{\star}+\omega^{2}\right) u=0\right\}$,

where we consider the topology induced by $\left[H^{1}(\Omega)\right]^{D}$.

Theorem 1. For any frequency $\omega \in \mathbb{R}$, the space $S_{\omega}(\hat{\Gamma})$ is dense in $\mathscr{H}_{\omega}$. 
$\omega=3.3648$
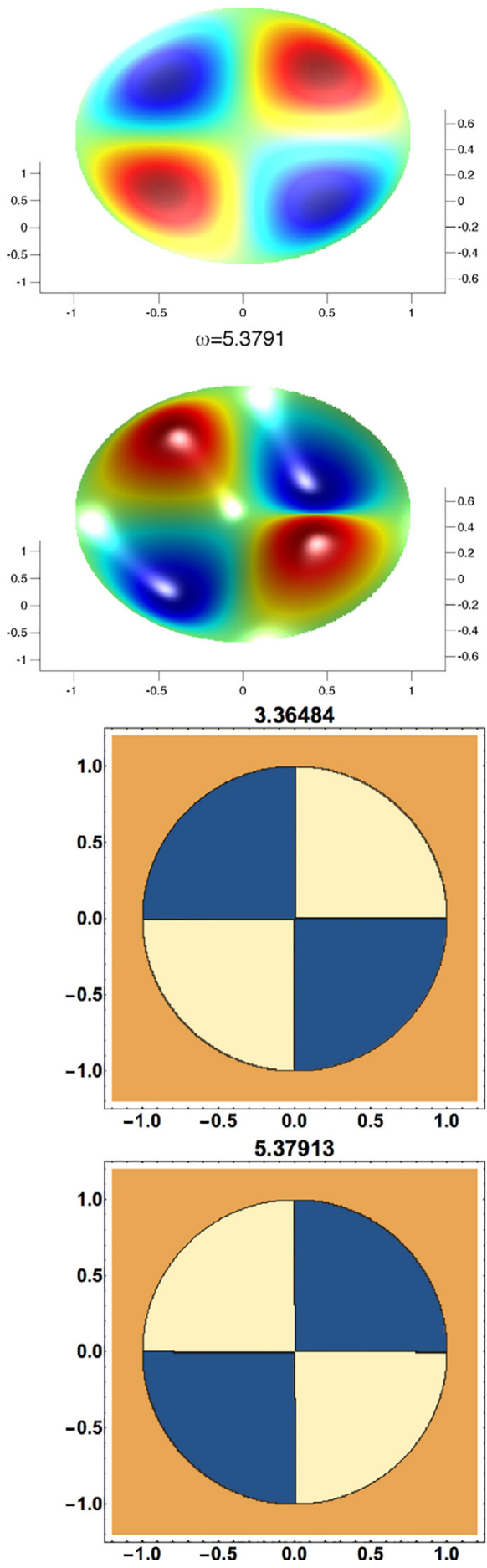

$\omega=3.8317$
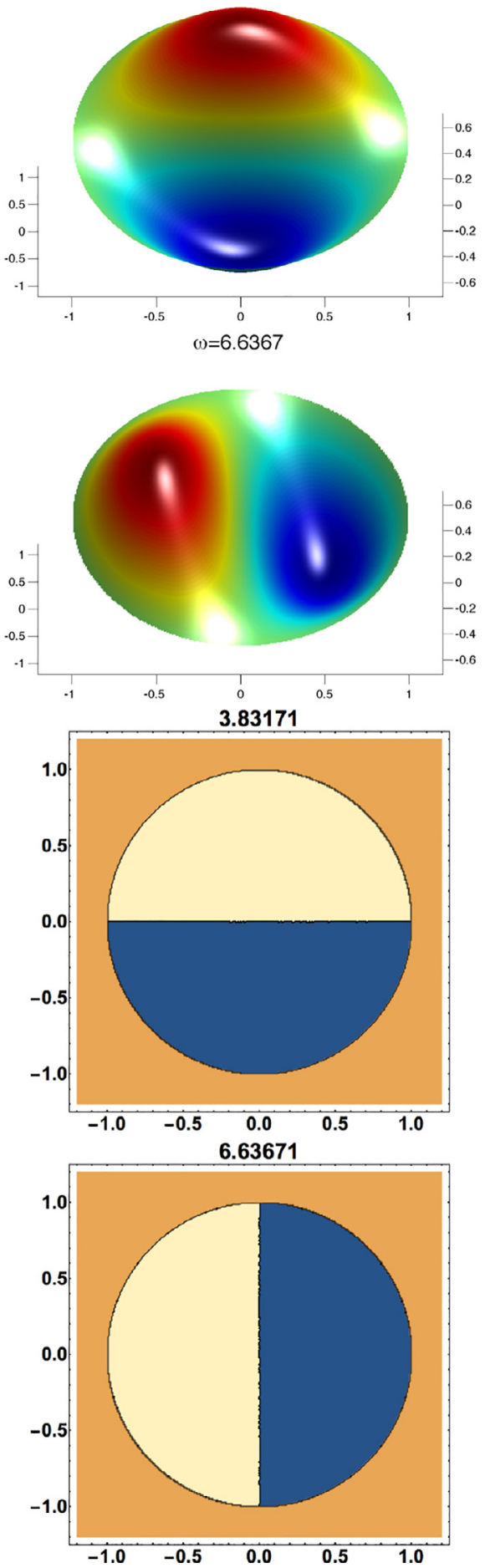

$\omega=5.2224$
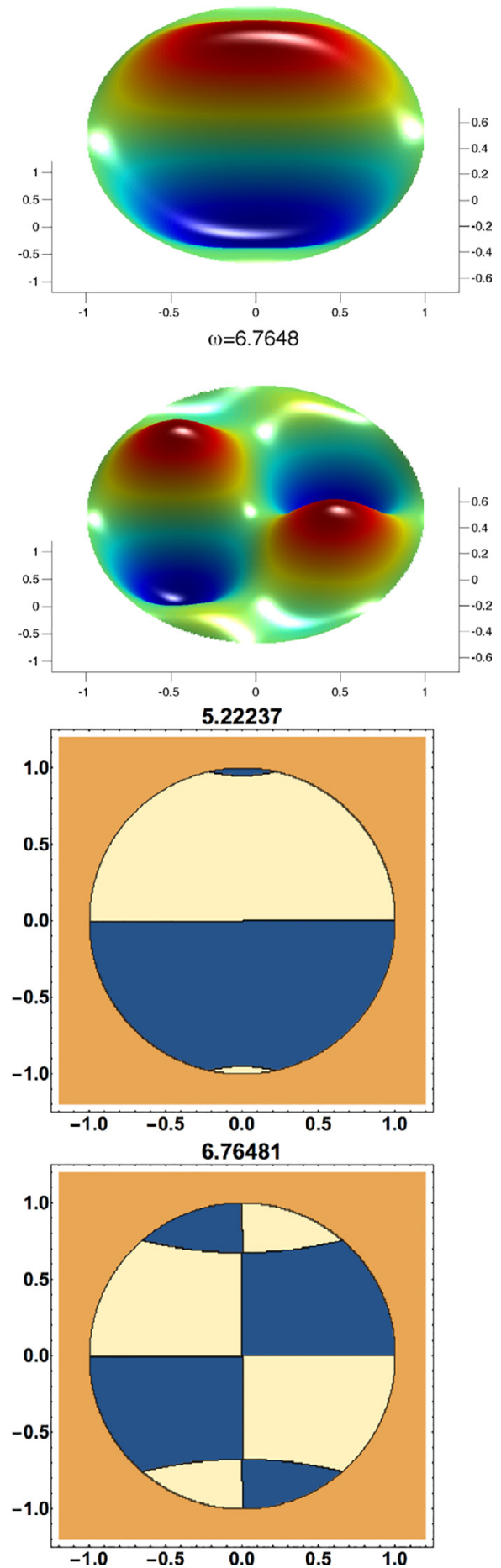

Fig. 5.5. The first components of the eigenmodes associated to the smallest six resonance frequencies of the unit disk and the corresponding nodal domains $\left(v=\frac{1}{4}\right)$.

Proof. Consider any $v \in \mathscr{H}_{\omega}$, and a function expressed by its Newtonian potential,

$u(y)=\left\langle\mathscr{G}_{\omega}(|\cdot-y|), \bar{v}\right\rangle_{\left[H^{1}(\Omega)\right]^{D} \times\left[H^{-1}(\Omega)\right]^{D}}=\int_{\Omega} \mathscr{G}_{\omega}(|x-y|) v(x) d x$.

We assume that $u(y)=0, \quad \forall y \in \hat{\Gamma}$ and we want to prove that this orthogonality condition in dual Banach spaces implies that $v \equiv 0$.

Since $v$ in only defined in $\Omega$ we extend this function by zero to the whole space, and the Newtonian potential may be seen as the convolution, $u=\mathscr{G}_{\omega} * \tilde{v}$, where $\tilde{v}$ represents the extended function. This implies
$-\left(\Delta^{\star}+\omega^{2}\right) u=\left(\Delta^{\star}+\omega^{2}\right)\left(\mathscr{G}_{\omega} * \tilde{v}\right)=(\delta \mathbf{I}) * \tilde{v}=\tilde{v}$,

in the whole space. In particular $\left(\Delta^{\star}+\omega^{2}\right) u=v$ in $\Omega$ and $\left(\Delta^{\star}+\omega^{2}\right) u=$ 0 in $\mathbb{R}^{D} \backslash \bar{\Omega}$.

As the convolution of $\tilde{v}$ with the fundamental solution also verifies the Sommerfeld radiation condition extended to the elastic problem, the well posedness of the exterior problem implies that the boundary condition $u=0$ on $\hat{\Gamma}$ will give a null solution $u=0$ in $\mathbb{R}^{D} \backslash \hat{\hat{\Omega}}$ which, by analytic continuation, is zero in $\mathbb{R}^{D} \backslash \bar{\Omega}$. 

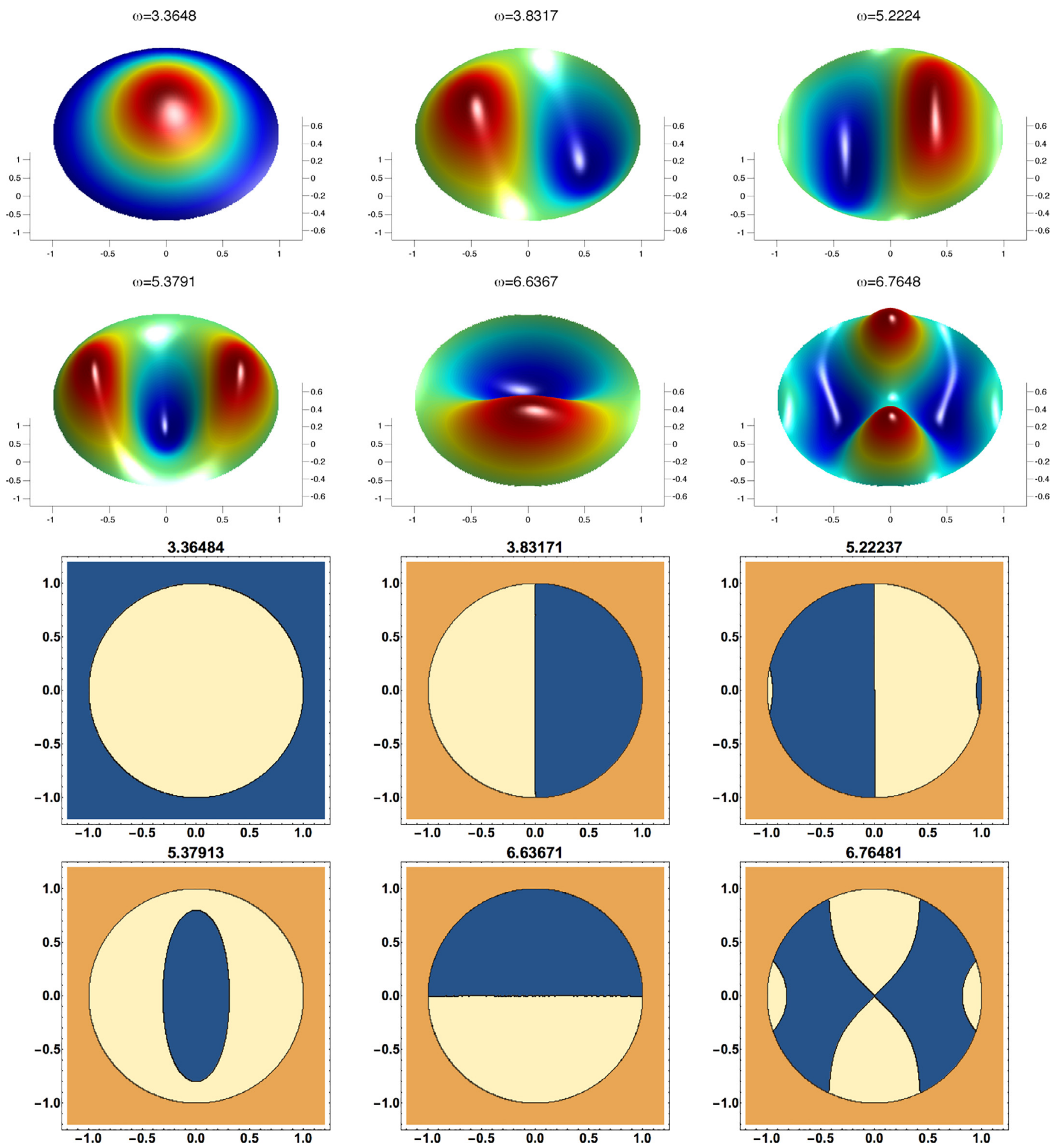

Fig. 5.6. The second components of the eigenmodes and nodal domains associated to Fig. 5.5.

Since the Newtonian potential has no jumps through the boundary, the null traces lead to null boundary conditions, and we have the interior elastic problem

$\begin{cases}\Delta^{\star} u+\omega^{2} u=v & \text { in } \Omega, \\ u=0 & \text { on } \Gamma, \\ \partial_{\mathrm{n}}^{\star} u=0 & \text { on } \Gamma,\end{cases}$

where $\partial_{\mathrm{n}}^{\star} u$ is the previously defined normal stress tensor.
Now, using the extended Green formula in elasticity, and since $\Delta^{\star} u=$ $v-\omega^{2} u, \Delta^{\star} v=-\omega^{2} v$,

$$
\begin{aligned}
0 & =\int_{\Gamma}\left(\left(\partial_{\mathrm{n}}^{\star} u\right) \cdot \bar{v}-\left(\overline{\partial_{\mathrm{n}}^{\star} v}\right) \cdot u\right)=\int_{\Omega}\left(\left(\Delta^{\star} u\right) \cdot \bar{v}-\left(\overline{\Delta^{\star} v}\right) \cdot u\right) \\
& =\int_{\Omega}\left(\left(v-\omega^{2} u\right) \cdot \bar{v}-\left(-\omega^{2} \bar{v}\right) \cdot u\right)=-\int_{\Omega} v \cdot \bar{v}=-\|v\|_{L^{2}(\Omega)}^{2}
\end{aligned}
$$

Since $\|v\|_{L^{2}(\Omega)}=0$, this implies $v \equiv 0$ and the density is proven, as it was shown that the null function is the only function in $\mathscr{H}_{\omega}$ that is orthogonal to all functions in $S_{\omega}(\hat{\Gamma})$. 


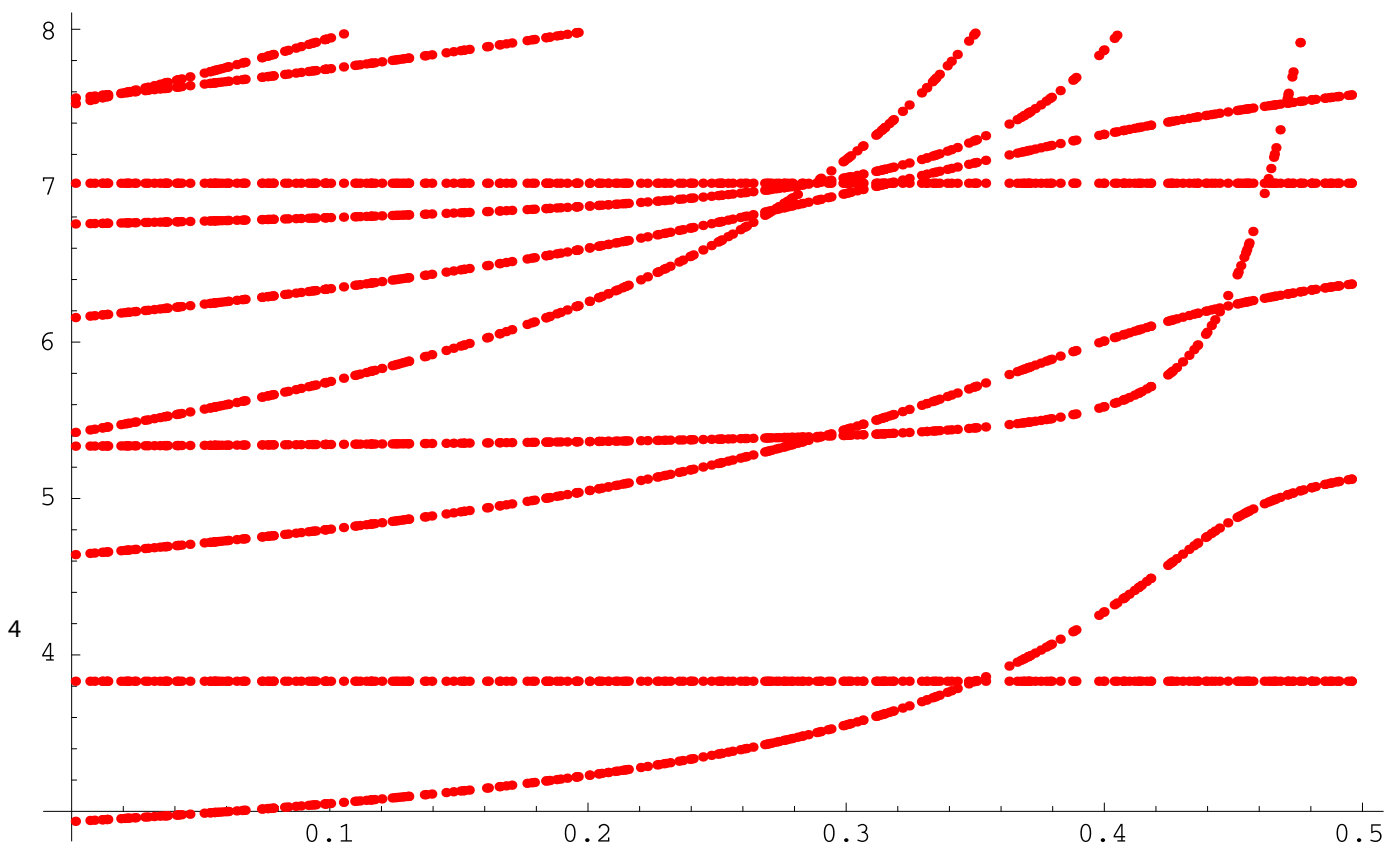

Fig. 5.7. The resonance frequencies of the unit disk, as a function of $v \in] 0,0.5[$.

Corollary 1. Let $\omega$ be a resonance frequency with an associated eigensolution $u \neq 0$ of the problem (2.4). Given any $\varepsilon>0$ there exist $y_{1}, \ldots, y_{m} \in \hat{\Gamma}$ and $a_{1}, \ldots, a_{m} \in \mathbb{C}^{D}$ such that an approximation

$u_{m}(x)=\sum_{j=1}^{m} \mathscr{G}_{\omega}\left(\left|x-y_{j}\right|\right) \boldsymbol{a}_{j}$

verifies

$\left\|u-u_{m}\right\|_{\left[H^{1}(\Omega)\right]^{D}}<\varepsilon$

Remark 1. From the previous result, given $\varepsilon>0$ any solution in (3.6) can be approximated by a sequence in (3.5) for sufficiently large $m$, such that

$\left\|u-u_{m}\right\|_{\left[H^{1}(\Omega)\right]^{D}}<\varepsilon$.

This holds for any type of boundary condition imposed in (2.4), since the eigensolution belongs to (3.6). However it should be noted that in this result the source points $y_{1}, \ldots, y_{m}$ are not given, they are unknown points in $\hat{\Gamma}$, but they might be approximated as close as we want, by taking $\hat{\Gamma}_{\mathrm{m}}$ sets, that in the limit are dense in $\hat{\Gamma}$.

Corollary 2. The frequency $\omega$ is a resonance frequency if and only if, given any $\varepsilon>0$ there is a solution $u_{m} \in S_{\omega}(\hat{\Gamma})$, with

$\left\|u_{m}\right\|_{\left[H^{1}(\Omega)\right]^{D}}=1$ and $\left\|B u_{m}\right\|_{\Gamma}<\varepsilon$.

(Here $\|.\|_{\Gamma}$ stands for the appropriate norm in the trace space; it is the $\left[H^{1 / 2}(\Gamma)\right]^{D}$ norm for the Dirichlet problem or the $\left[H^{-1 / 2}(\Gamma) / \mathbb{R}\right]^{D}$ norm for Neumann boundary conditions)

Proof. If $\omega$ is a resonance frequency there is an eigensolution $u \neq 0$ with $B u=0$ on $\Gamma$ and $\|u\|_{\left[H^{1}(\Omega)\right]^{D}}=1$. With $\varepsilon_{1}>0$, from Corollary 1 there exists $v_{m} \in S_{\omega}(\hat{\Gamma})$ such that $\left\|u-v_{m}\right\|_{\left[H^{1}(\Omega)\right]^{D}}<\varepsilon_{1}$.

The trace theorem implies that there exists $C>0$ such that $\|B w\|_{\Gamma} \leq$ $C\|w\|_{\left[H^{1}(\Omega)\right]^{D}}$ and taking $w=u-v_{m}$ this gives

$\left\|B v_{m}\right\|_{\Gamma}=\left\|\mathcal{B} u-B v_{m}\right\|_{\Gamma} \leq C\left\|u-v_{m}\right\|_{\left[H^{1}(\Omega)\right]^{D}}<C \varepsilon_{1}$.

It is enough to take $u_{m}=v_{m} /\left\|v_{m}\right\|_{\left[H^{1}(\Omega)\right]^{D}}$ with unitary norm to conclude

$\left\|B u_{m}\right\|_{\Gamma}<C \varepsilon_{1} /\left\|v_{m}\right\|_{\left[H^{1}(\Omega)\right]^{D}} \leq C \frac{\varepsilon_{1}}{1-\varepsilon_{1}}=\varepsilon$

taking $\varepsilon_{1}=\frac{\varepsilon}{\varepsilon+C}$, for any given $\varepsilon>0$.
On the other hand, assume that $\left\|B u_{m}\right\|_{\Gamma} \rightarrow 0$ with $\left\|u_{m}\right\|_{\left[H^{1}(\Omega)\right]^{D}}=1$. Due to continuity, $u_{m} \rightarrow u$ with $B u=0$ and $\|u\|_{\left[H^{1}(\Omega)\right]^{D}}=1 \neq 0$, and this means that $\omega$ is a resonance frequency and $u$ is an associated eigenmode.

Remark 2. This corollary shows that by searching solutions in $S_{\omega}\left(\hat{\Gamma}_{m}\right)$ that verify the homogeneous boundary condition (up to $\varepsilon$ ) and that are not null, we obtain all the resonance frequencies spectrum.

\section{Determination of resonance frequencies and eigenmodes with} the MFS

We now consider an artificial set $\hat{\Gamma}=\partial \hat{\Omega}$ that surrounds $\Gamma=\partial \Omega$ by taking $\bar{\Omega} \subset \hat{\Omega}$, and consider the source points $y_{1}, \ldots, y_{m} \in \hat{\Gamma}$. We also consider a set of collocation points $x_{1}, \ldots, x_{n} \in \Gamma$ and define the collocation matrix

$\mathbf{M}(\omega)=\left[\begin{array}{ccc}{\left[\mathcal{B} \mathscr{G}_{\omega}\left(\left|x_{1}-y_{1}\right|\right)\right]_{D \times D}} & \cdots & {\left[\mathcal{B} \mathscr{G}_{\omega}\left(\left|x_{1}-y_{m}\right|\right)\right]_{D \times D}} \\ \vdots & \ddots & \vdots \\ {\left[\mathcal{B} \mathscr{G}_{\omega}\left(\left|x_{n}-y_{1}\right|\right)\right]_{D \times D}} & \cdots & {\left[\mathcal{B} \mathscr{G}_{\omega}\left(\left|x_{n}-y_{m}\right|\right)\right]_{D \times D}}\end{array}\right]$,

with $m=n$. In this work we will follow the choice for the collocation and source points introduced in [2] (other recent approaches to avoid a ficticious boundary were considered in $[12,23])$. We start by distributing $m$ points $x_{1}, \ldots, x_{m}$ almost uniformly on the boundary $\Gamma$ and for each of these points we define the corresponding source point

$y_{k}=x_{k}+\alpha \mathrm{n}_{k}, k=1, \ldots, m$,

where $\mathrm{n}_{k}$ is the unitary outward normal vector at the point $x_{k}$ and $\alpha$ is a prescribed positive parameter.

\subsection{Resonance frequencies}

To calculate the resonance frequencies we will search for the frequencies $\omega$ such that this system has non trivial solutions. This can be done, for instance, by calculating the frequencies for which the matrix $\mathbf{M}(\omega)$ is not invertible, i.e.

$\operatorname{det} \mathbf{M}(\omega)=0$ 

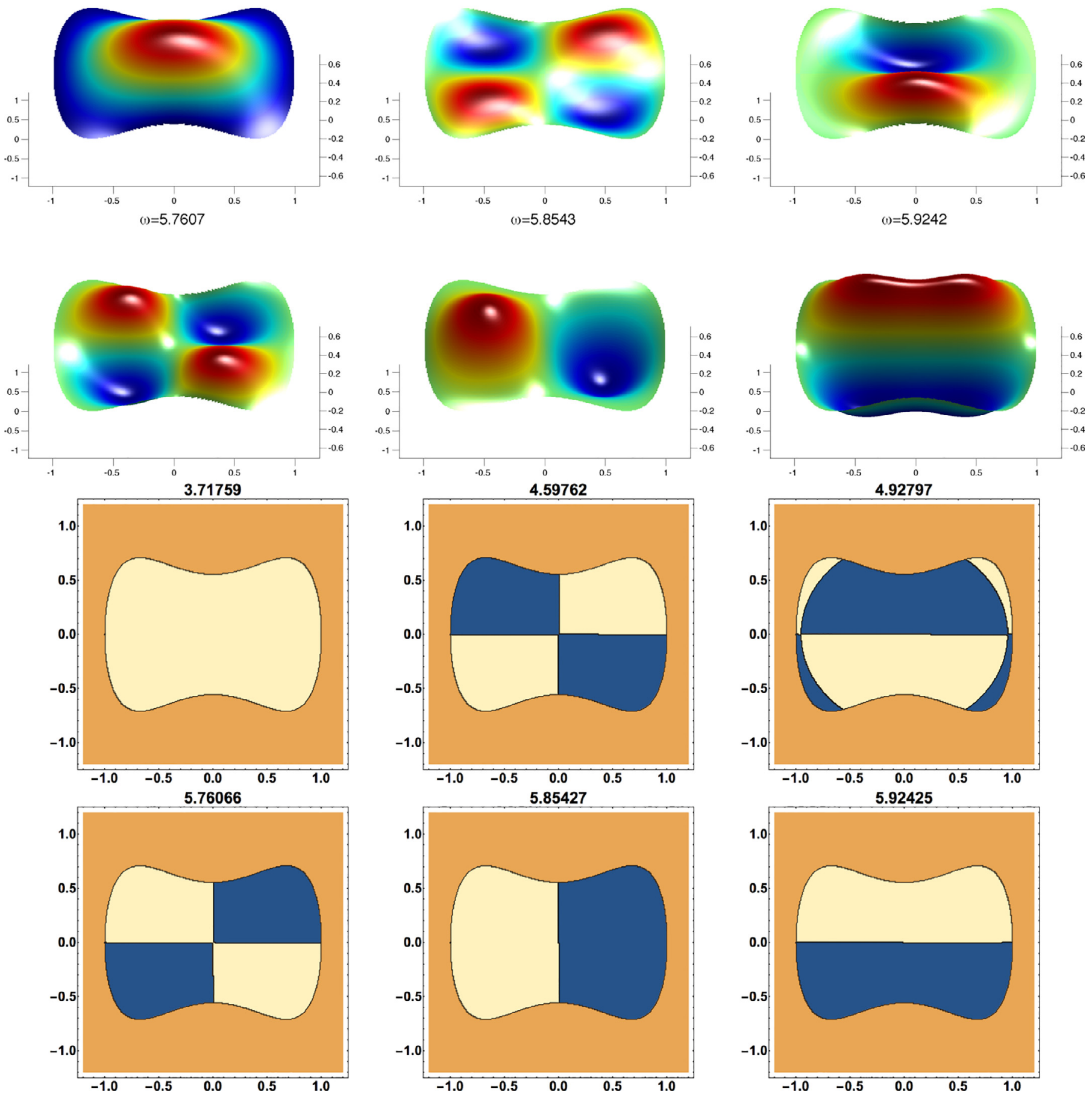

Fig. 5.8. Plots of the first components of eigenmodes corresponding to the smallest six resonance frequencies of the domain $\Omega_{1}\left(v=\frac{1}{4}\right)$.

or equivalently the frequencies for which

$$
g(\omega):=\log (|\operatorname{det} \mathbf{M}(\omega)|)
$$

has a singularity.

Thus, the calculation of each resonance frequency implies the solution of a single-variable nonlinear minimization problem that can be performed, for example, using a direct search method. In this work we used the golden ratio search, as was done in [2], for the scalar acoustic problem.
Remark 3. To avoid the extremely low values of the determinant, its logarithm is calculated within LU factorization, since

$\log (\operatorname{det} \mathbf{M}(\omega))=\log (\operatorname{det} \mathbf{U})=\log \left(u_{11} \cdots u_{N N}\right)=\log \left(u_{11}\right)+\cdots+\log \left(u_{N N}\right)$

with $\mathbf{M}(\omega)=\mathbf{L} \mathbf{U}$ and $N=m D$. Thus, the calculation of $u_{11} \cdots u_{N N}$ is avoided and only a sum of logarithms is considered. Another possibility to circumvent the ill conditioning of MFS matrices is to consider a subspace angle technique, as proposed in [10]. 

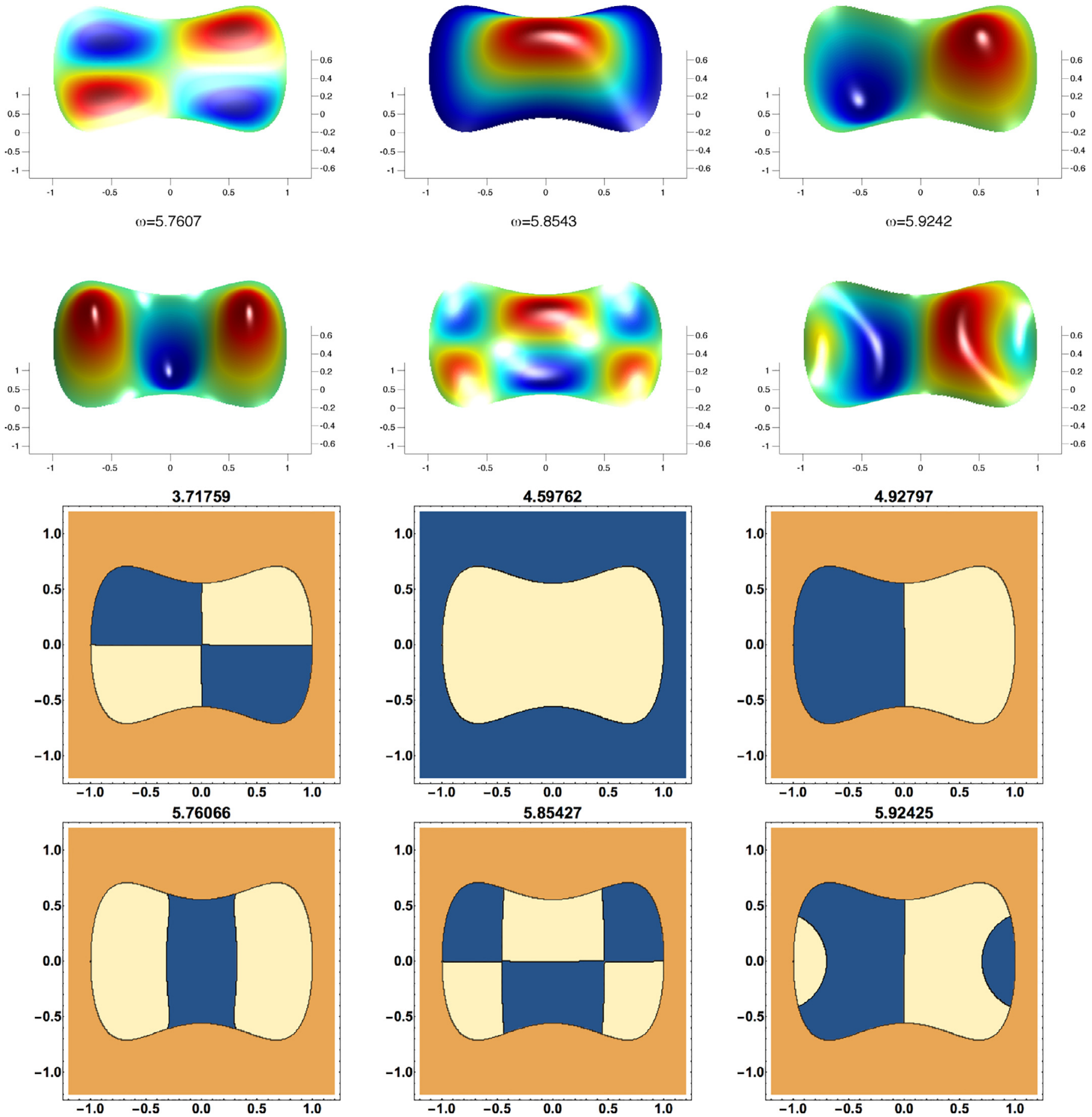

Fig. 5.9. Plots of the second components of the eigenmodes and nodal domains associated to Fig. 5.8.

\subsection{Eigenmodes}

After calculating an approximate resonance frequency $\tilde{\omega}$ we are interested in determining an associated eigenmode. In elastodynamics we are also interested in excluding the null solution from the possible solutions of the system that give the approximate eigenmode.

We consider two methods:

(i) In [2] we obtained the eigenmodes by choosing $m+1$ point sources and collocation points, $m$ points on the boundary and an extra collocation point $x_{m+1}$ inside the domain. To exclude the null solution from the possible solutions we calculated the approximate eigenmode $\tilde{u}$ by imposing that $\tilde{u}\left(x_{m+1}\right)=1$.

(ii) Another possibility is to impose in the system that, for example, one of the components of the vector $\boldsymbol{a}_{m}$ is equal to one.

The procedure (ii) has some advantages when compared with (i).

Instead of a $(m+1) \times(m+1)$ system we have a $m \times m$ system, but more importantly this avoids the "artificial" choice of the extra collocation point. As stated, the extra collocation point must be placed 


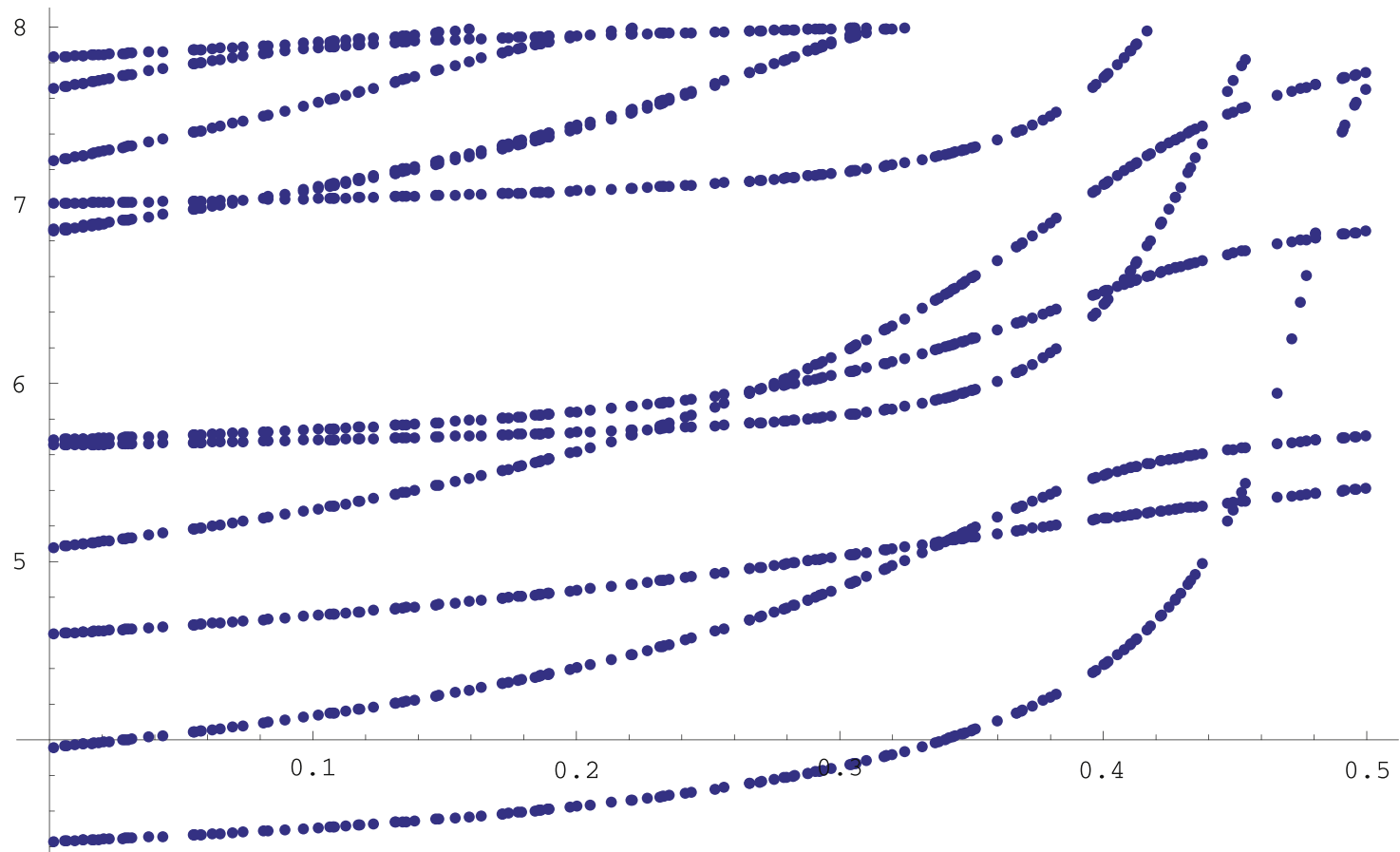

Fig. 5.10. The resonance frequencies of the domain $\Omega_{1}$, as a function of $\left.v \in\right] 0,0.5[$.

outside the nodal lines, and specially for high frequencies, the location must be carefully chosen and this disadvantage is avoided imposing, for example, that the second component of the coefficient $\boldsymbol{a}_{m}=\left(a_{m}^{1}, a_{m}^{2}\right)$ is equal to one.

For instance, with $D=2$, given an approximate eigenfrequency $\tilde{\omega}$, and using the notation

$\mathbb{G}_{i, j}=\left[\mathscr{G}_{\tilde{\omega}}\left(\left|x_{i}-y_{j}\right|\right)\right]_{2 \times 2}$,

the system becomes

$\left.\left[\begin{array}{ccc}\mathbb{G}_{1,1} & \cdots & \mathbb{G}_{1, N} \\ \cdots & \cdots & \cdots \\ \left(\begin{array}{cc}\mathbb{G}_{m, 1}^{1,1} & \mathbb{G}_{m, 1}^{1,2} \\ 0 & 0\end{array}\right) & \cdots & \left(\begin{array}{cc}\mathbb{G}_{m, m}^{1,1} & \mathbb{G}_{m, m}^{1,2} \\ 0 & 1\end{array}\right)\end{array}\right]\left[\begin{array}{c}\boldsymbol{a}_{1} \\ \cdots \\ \left(\begin{array}{c}a_{m}^{1} \\ a_{m}^{2}\end{array}\right)\end{array}\right]=\left[\begin{array}{c}(0)_{2 \times 1} \\ \ldots \\ 0 \\ 1\end{array}\right)\right]$

After solving this last system we substitute the coefficients in the linear combination of fundamental solutions (2.5) to obtain the associated eigenmode.

\section{Numerical simulations}

In this section we will present some numerical results obtained with the proposed algorithm. All the results will be presented in terms of the Poisson coefficient $v$ that can be defined in terms of the Lamé parameters by

$$
v=\frac{\lambda}{2(\lambda+\mu)} \text {. }
$$

It is important to observe that in the elastic case there is a change of the resonance frequencies with respect to the material properties given by $v$. In applications this change may be of importance in checking the possible failure of a shell in a structure due to natural resonance phenomena.

We start with a validation of our numerical algorithm in the case of the unit disk, for which we know the exact solution.

Considering $v=0.25$, in Fig. 5.1 we plot $g(\omega)$, obtained with $m=30$, $\alpha=0.5$. We also marked with a dashed line the location of the exact eigenfrequencies. We can see that the plot presents a singularity at each one of the eigenfrequencies.
Table 1

Absolute errors of the former three eigenfrequencies of the disk with $v=0.25$.

\begin{tabular}{llll}
\hline$m$ & Abs. error $\left(\omega_{1}\right)$ & Abs. error $\left(\omega_{2}\right)$ & Abs. error $\left(\omega_{3}\right)$ \\
\hline 30 & $6.69 \times 10^{-6}$ & $9.69 \times 10^{-7}$ & $6.60 \times 10^{-6}$ \\
50 & $1.80 \times 10^{-9}$ & $3.03 \times 10^{-10}$ & $1.69 \times 10^{-9}$ \\
70 & $5.28 \times 10^{-13}$ & $8.70 \times 10^{-14}$ & $4.62 \times 10^{-13}$ \\
\hline
\end{tabular}

In Table 1 we show results for the absolute errors obtained with different numbers of points, and we can see that the convergence is very fast.

Next, we show some results regarding the absolute errors obtained for the first resonance frequency of the unit disk by varying the parameter $\alpha$.

In the first plot of Fig. 5.2 we show the absolute errors obtained with $m=20,30,40,50,60$, for $\alpha \in[0.1,3]$.

In general, for simple geometries, the error decreases when we increase the parameter $\alpha$, by moving the auxiliary boundary away from the physical boundary $\Gamma$, as illustrated in Fig. 5.2-left.

In the right plot of the same figure, we show results for the condition number

$\operatorname{cond}(\mathbf{M})=\frac{\max _{i}\left|\lambda_{i}(\mathbf{M}(\tilde{\omega}))\right|}{\min _{i}\left|\lambda_{i}(\mathbf{M}(\tilde{\omega}))\right|}$

where $\lambda_{i}(\mathbf{M}(\tilde{\omega}))$ are the eigenvalues of the matrix $\mathbf{M}(\tilde{\omega})$.

For instance, following the results obtained with $m=60$, we see that the absolute errors decrease when we increase the value of $\alpha$ until a value $\alpha^{*}$ approximately equal to 0.8 is reached. For $\alpha>\alpha^{*}$ the convergence curve is broken due to the ill conditioning of the matrix.

This problem could be circumvented using the MFS-QR technique (cf. [8]).

Now we test the numerical method with other choices for the source points.

We consider the domain which is the intersection of two unit circles centered at the points $(0,1 / 2)$ and $(0,-1 / 2)$. In Fig. 5.3 we plot 80 col- 

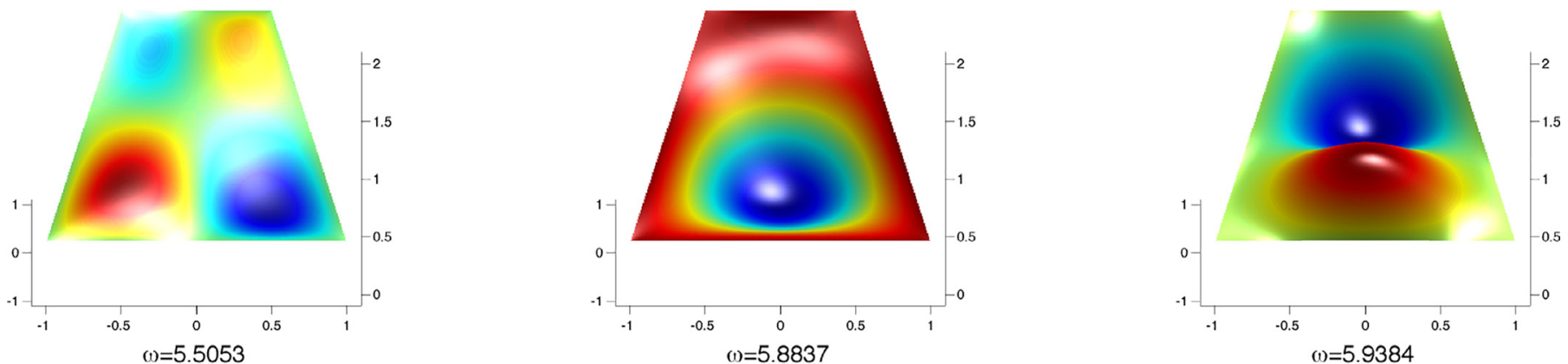
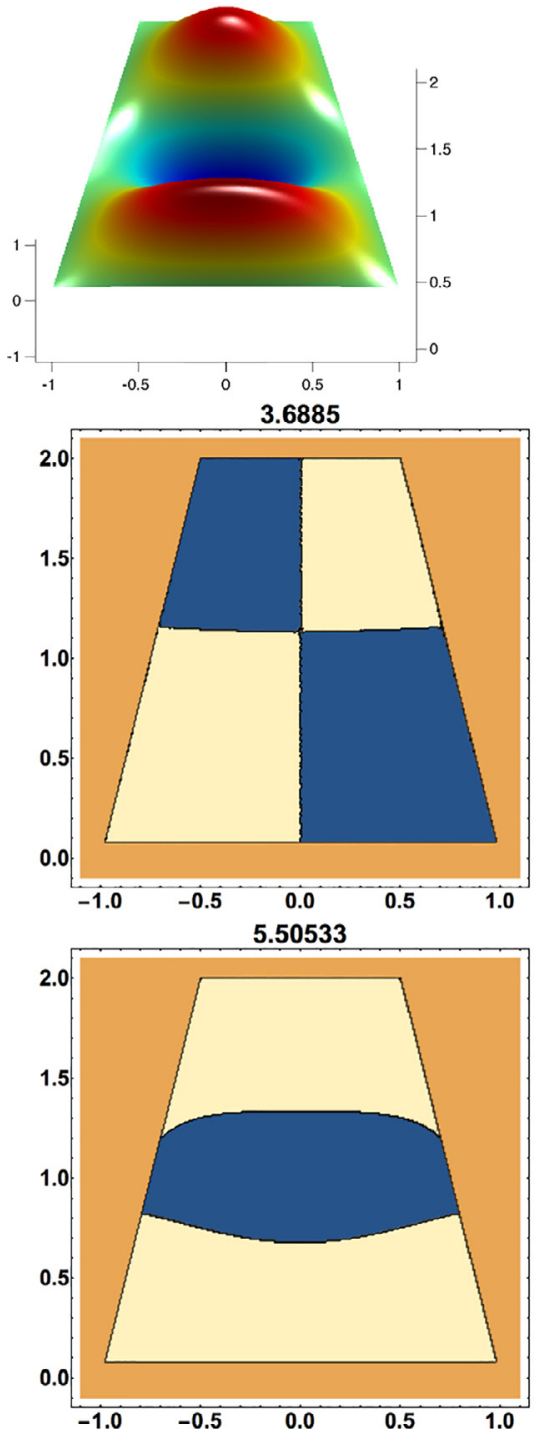
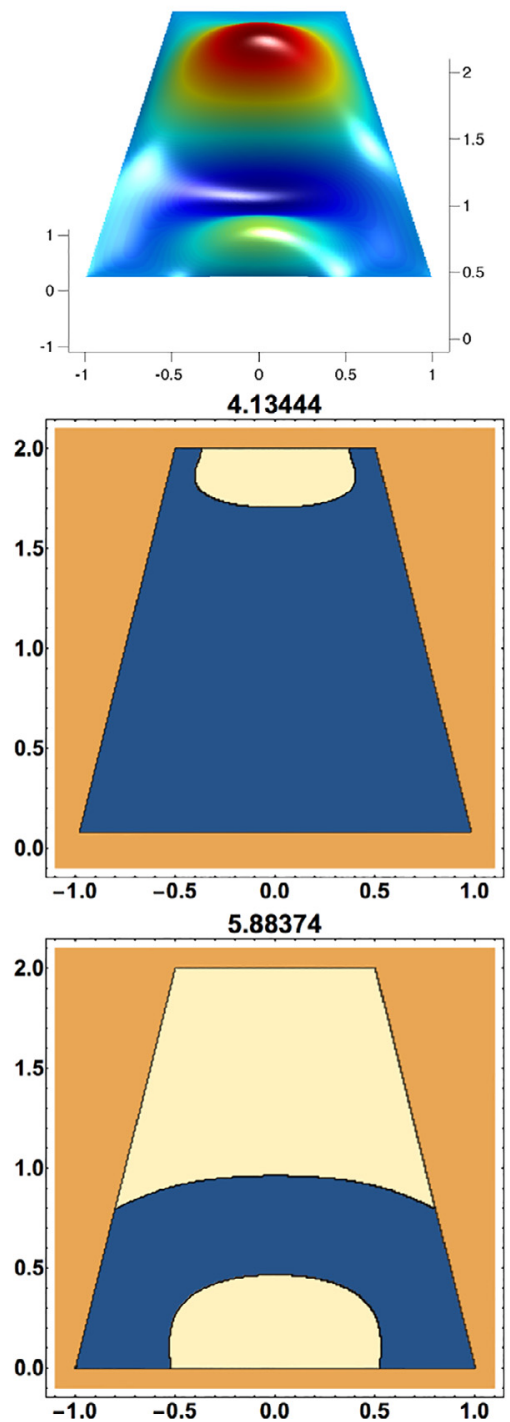
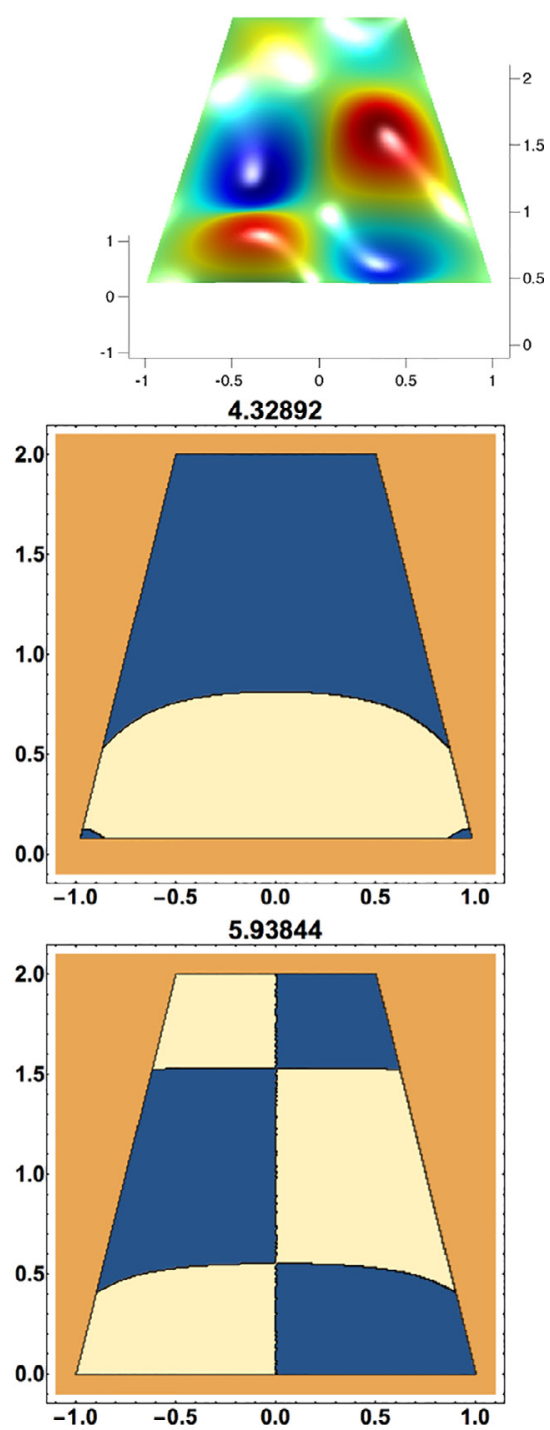

Fig. 5.11. The first components of the eigenmodes associated to the smallest six resonance frequencies of the trapezoid defined through the vertices $(-1,0),(1,0)$, $\left(\frac{1}{2}, 2\right)$ and $\left(-\frac{1}{2}, 2\right)$ and the corresponding nodal domains $\left(v=\frac{1}{6}\right)$.

location points and three possible choices for the location of the pointsources. In the first plot we distribute the point sources on the boundary of an elipse with axes lengths 2.4 and 1.6, in the second we define the point-sources as an "expansion" of the collocation points $\left(y_{i}=1.7 x_{i}\right)$.

In the last case we consider the source points defined by (4.2) with $\alpha=0.4$. In Fig. 5.4 we plot $g(\omega)$ for these three choices of source points.
We can observe that in the first two cases we have large numerical errors, due the ill conditioning of the matrix and we are not able to locate the singularities which correspond to the numerical approximations for the eigenfrequencies. In the last plot, obtained with the choice we proposed, the errors are much smaller and this allows to locate the eigenfrequencies. 

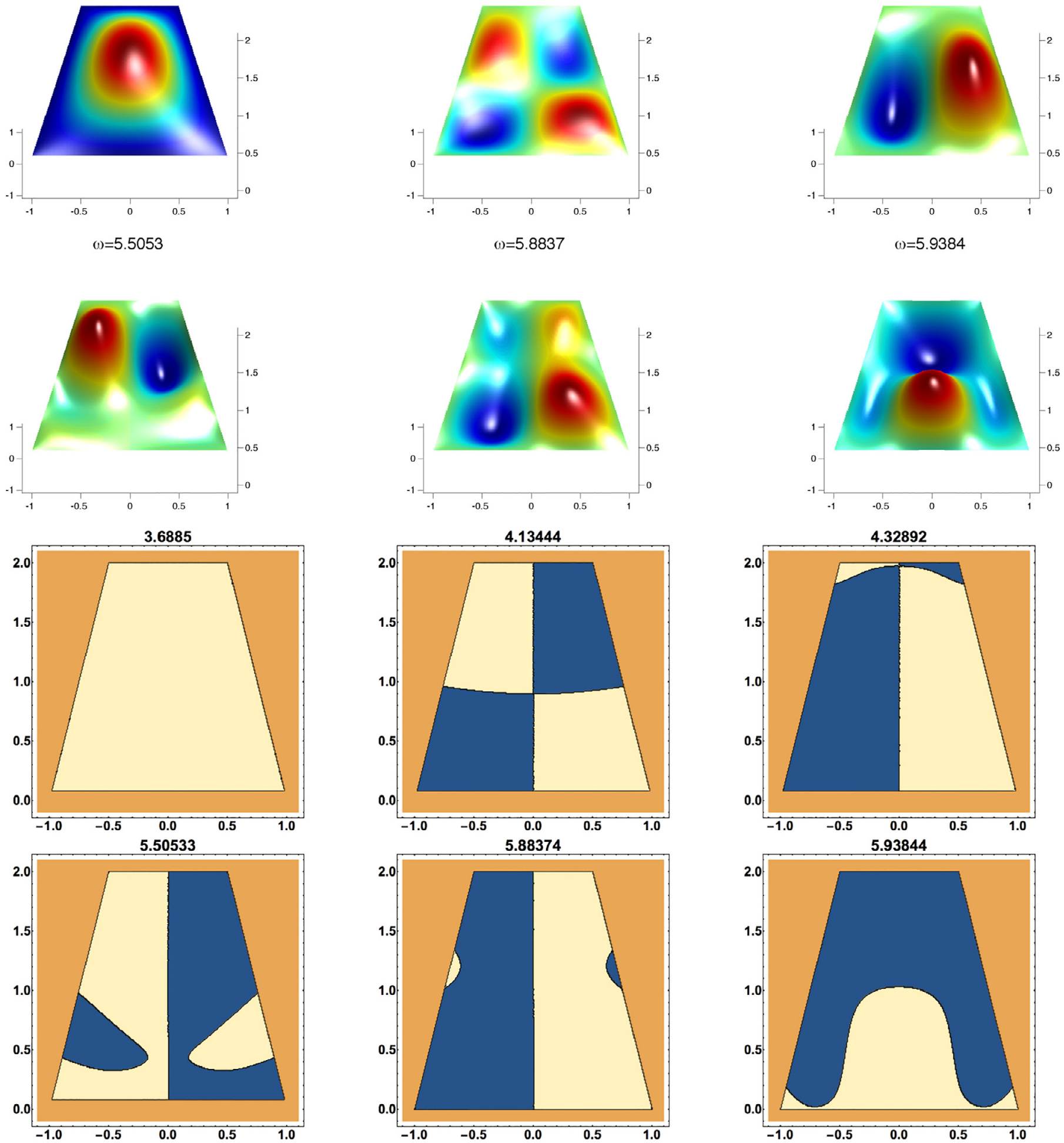

Fig. 5.12. The second components of the eigenmodes and nodal domains associated to Fig. 5.11.

\subsection{Unit disk}

In Figs. 5.5 and 5.6 we plot, respectively, the first and second components of the eigenmodes and the corresponding nodal domains associated to the smallest six eigenfrequencies of the unit disk (with $v=\frac{1}{4}$ ).
As in the acoustic problem, we could expect that the first eigenmode would be always simple (for connected domains) and would not present nodal lines cutting the disk in four parts, as we may see in Fig. 5.5 for $\omega=3.36484 \ldots$ However this does not happen for the first component and it only happens for the second component, as we may see in Fig. 5.6. 
This may be verified considering the calculation of the multiplicities of the resonance frequencies. Indeed, we have several multiple eigenfrequencies. For instance, if we denote by $\omega_{i}$, the $i$-th eigenfrequency, counting with multiplicities, in this case of $v=\frac{1}{4}$ we have

$0<\omega_{1}=\omega_{2}<\omega_{3}<\omega_{4}=\omega_{5}<\omega_{6}=\omega_{7}<\omega_{8}<\omega_{9}=\omega_{10}<\cdots$

Thus, in the case of eigenfrequencies with multiplicity two, we are just presenting one possible eigenmode.

We observe that the plots of the nodal lines show some coincidence with the nodal lines that are present in the acoustic case, but some of them are more complex, which was also expected.

We are interested in knowing how the change of the Poisson factor affects the resonance frequencies. This was plotted in Fig. 5.7. In that picture we plot the resonance frequencies of the unit disk, as a function of $v \in] 0,0.5[$. We can see that the plots show continuous lines that flow with the increase of the Poisson coefficient, with some possible intersections. This suggests a differentiable continuation of the resonance frequencies with $v$, but that does not respect the ordering of the magnitude.

\subsection{Non trivial domain (1)}

Next, we show some numerical results obtained for a non trivial domain $\Omega_{1}$ whose boundary is defined by

$\partial \Omega_{1}=\left\{\left(\cos (t), \sin (t)+\frac{\sin (2 t)}{3}\right): 0 \leq t<2 \pi\right\}$.

In Figs. 5.8 and 5.9 we plot (respectively) the first and second components of the eigenmodes associated to the first six eigenfrequencies of the domain $\Omega_{1}$ and the corresponding nodal domains, obtained for $v=\frac{1}{4}$. Similar patterns to the ones obtained in the case of the disk are found here.

Again we are interested in changing the Poisson coefficient, and in Fig. 5.10 we plot the resonance frequencies of the domain $\Omega_{1}$ in the interval $[3,8]$, as a function of $v$. We may see that, despite some expected differences, the plots also show a similar pattern to the one obtained in the case of the unit disk, namely in the continuous evolution of the resonance frequencies with respect to the Poisson ratio.

\subsection{Non trivial domain (2)}

Finally, we consider a domain with a non-smooth boundary - a trapezoid with vertices at the points $(-1,0),(1,0),\left(\frac{1}{2}, 2\right)$ and $\left(-\frac{1}{2}, 2\right)$. Numerical results are presented with a different Poisson coefficient, $v=\frac{1}{6}$.

Figs. 5.11 and 5.12 show the first and second components of the eigenmodes associated to the smallest six resonance frequencies of the trapezoid and the corresponding nodal domains. These illustrate the good performance of the method, even for non-smooth domains. However the accuracy of the MFS when applied to more complicated nonsmooth regions, such as domains with reentrant corners, is not so good, as the solution is not so smooth. An interesting topic that is being considered under current research is to extend the approach considered in [9] to the elastic case.

\section{Conclusions}

In this paper we apply the MFS for the calculation of elastic resonance frequencies and the associated eigenmodes. To the best of our knowledge the theoretical and computational aspects of this subject have not been previously investigated.

The paper presents a theoretical part that is valid for dimensions $D=2$ or 3 , where density results were derived, and a numerical part that was only implemented in 2D. We do not expect major difficulties in 3D applications, except in the increase of complexity associated with the increase in dimension.
In this work we only considered the computation of resonance frequencies for the Dirichlet problem, but current research is being carried for other boundary conditions, and also for domains that are not simply connected, presenting different boundary conditions, and where techniques (like in $[17,30]$ ) have to be considered to avoid spurious eigenfrequencies.

The MFS yields excellent results, achieving double precision accuracy for regular shapes, such as the disk and some other non trivial smooth domains, such as the one presented in Section 5.2. This is a huge advantage with respect to other mesh-type methods, such as the finite element method, where extremely large triangulations would have to be considered just to get single precision results.

The performance for non-smooth domains is more delicate. In Section 5.3 we presented a trapezoidal domain for which singleprecision accuracy was obtained, but we expect higher difficulties for domains with reentrant corners. The improvement of the results for these irregular shapes is under current research, extending the approach considered in [9] to the elastic case.

\section{Acknowledgments}

The financial support received from the Fundação para a Ciência e a Tecnologia (FCT), through the program "Investigador FCT" with reference IF/00177/2013 and the scientific projects UID/Multi/ 04621/2013 and PTDC/MAT- CAL/4334/2014 is gratefully acknowledged.

\section{References}

[1] Alves CJS. Numerical simulations on resonance poles - the trapping case of two plane cracks. J Comput Appl Math 1999;111:267-79.

[2] Alves CJS, Antunes PRS. The method of fundamental solutions applied to the calculation of eigenfrequencies and eigenmodes of $2 \mathrm{~d}$ simply connected shapes. Comput Mater Continua 2005;2(4):251-66.

[3] Alves CJS, Antunes PRS. The method of fundamental solutions applied to the calculation of eigensolutions for 2d plates. Int J Num Meth Eng 2009;77:177-94.

[4] Alves CJS, Antunes PRS. The method of fundamental solutions applied to some inverse eigenproblems. SIAM J Sci Comput 2013;35:A1689-708.

[5] Alves CJS, Martins NFM. The direct method of fundamental solutions and the inverse Kirsch-Kress method for the reconstruction of elastic inclusions or cavities. J Integr Eq Appl 2009;21:153-78.

[6] Alves CJS, Martins NFM, Roberty NC. Identification and reconstruction of elastic body forces. Inverse Probl 2014;30:055015.

[7] Alves CJS, Martins NFM, Valtchev SS. Extending the method of fundamental solutions to non-homogeneous elastic wave problems. Appl Numer Math 2017; 115:299-313.

[8] Antunes PRS. A numerical algorithm to reduce ill-conditioning in meshless methods for the Helmholtz equation. Numer Algor 2018;79:879-97.

[9] Antunes PRS, Valtchev SS. A meshfree numerical method for acoustic wave propagation problems in planar domains with corners and cracks. J Comput Appl Math 2010;234:2646-62.

[10] Betcke T, Trefethen LN. Reviving the method of particular solutions. SIAM Rev 2003;47:469-91

[11] Fairweather G, Karageorghis A, Martin PA. The method of fundamental solutions for scattering and radiation problems. Eng Anal Bound Elem 2003;27:759-69.

[12] Fu Z, Chen W, Wen P, Zhang C. Singular boundary method for wave propagation analysis in periodic structures. J Sound Vibr 2018;425:170-88.

[13] Glushkov EV, Glushkova NV, Eremin AA, Lammering R. Guided wave propagation and diffraction in plates with obstacles: resonance transmission and trapping mode effects. Phys Procedia 2015;70:447-50.

[14] Godinho L, Tadeu A, Mendes PA. Wave propagation around thin structures using the MFS. Comput Mater Continua 2007;5:117-27.

[15] Godinho L, Amado-Mendes P, Pereira A. A hybrid analytical-numerical model based on the method of fundamental solutions for the analysis of sound scattering by buried shell structures. Math Probl Eng 2011:22. 710623

[16] Chen JT, Chen IL, Chen KH, Lee YT, Yeh YT. A meshless method for free vibration analysis of circular and rectangular clamped plates using radial basis function. Eng Anal Bound Elem 2004;28:535-45.

[17] Chen JT, Chen IL, Lee YT. Eigensolutions of multiply connected membranes using the method of fundamental solutions. Eng Anal Bound Elem 2005;29:166-74.

[18] Karageorghis A. The method of fundamental solutions for the calculation of the eigenvalues of the Helmholtz equation. Appl Math Lett 2001;14:837-42.

[19] Karageorghis A, Fairweather G. The method of fundamental solutions for axisymmetric elasticity problems. Comput Mech 2000;25:524-32.

[20] Kitahara M. Boundary integral equation methods in eigenvalue problems of elastodynamics and thin plates. Amsterdam: Elsevier; 1985.

[21] Kupradze V.D., et al. Three-dimensional problems of the mathematical theory of elasticity and thermoelasticity. 1979. North-Holland, Amsterdam. 
[22] Lee S. The use of equivalent source method in computational acoustics. J Comput Acoust 2017;25:19. 1630001.

23] Lin J, Zhang C, Sun LL, Lu J. Simulation of seismic wave scattering by embedded cavities in an elastic half-plane using the novel singular boundary method. Adv Appl Math Mech 2018;10:322-42.

[24] Lindsay AE, Quaife B, Wendelberger L. A boundary integral equation method for mode elimination and vibration confinement in thin plates with clamped points. Adv Comput Math 2018;44:1249-73.

[25] Marin L, Lesnic D. The method of fundamental solutions for the cauchy problem in two-dimensional linear elasticity. Int J Solids Struct 2004;41:3425-38.

[26] McLean W. Strongly elliptic systems and boundary integral equations. Cambridge University Press; 2000.
[27] Poullikkas A, Karageorghis A, Georgiou G. The method of fundamental solutions for three-dimensional elastostatics problems. Comput Struct 2002;80:365-70

[28] Reutskiy SY. The method of fundamental solutions for problems of free vibrations of plates. Eng Anal Bound Elem 2007;31:10-21.

[29] Smyrlis YS. Mathematical foundation of the MFS for certain elliptic systems in linear elasticity. Numer Math 2009;112:319-40.

[30] Tsai CC, Young DL, Chen CW, Fan CM. The method of fundamental solution for eigenproblems in domains with and without interior holes. Proc R Soc A 2006;462:1443-66.

[31] Wu CS, Young DL, Fan CM. Frequency response analyses in vibroacoustics using the method of fundamental solutions. Comput Mech 2011;47:519-33. 